\title{
Platelet function in patients with obstructive sleep apnoea syndrome
}

\author{
B.M. Sanner*, M. Konermann*, M. Tepel*, J. Groetz*, C. Mummenhoff*, W. Zidek*
}

Platelet function in patients with obstructive sleep apnoea syndrome. B.M. Sanner, M. Konermann, M. Tepel, J. Groetz, C. Mummenhoff, W. Zidek. (C)ERS Journals Ltd 2000. ABSTRACT: Patients with obstructive sleep apnoea syndrome (OSAS) are subject to an increased cardiovascular morbidity including myocardial infarction and stroke. Platelets play an important role in the pathogenesis and triggering of acute cardiovascular syndromes. So far, the influence of OSAS on platelet function is not fully understood.

Platelet aggregability to epinephrine, collagen, arachidonic acid, and adenosine diphosphate in vitro was measured in 17 consecutive male patients $(53.0 \pm 2.1$ yrs) with polysomnographically verified OSAS and compared with that of 15 male controls (50.1 $\pm 3.6 \mathrm{yrs})$ at 20:00 $\mathrm{h}, 24: 00 \mathrm{~h}$, and 06:00 $\mathrm{h}$. In addition, the long-term effects of continuous positive airway pressure (CPAP) therapy on platelet aggregability was assessed after 6 months.

Platelet aggregation in vitro induced by epinephrine showed a slight increase overnight in the untreated OSAS patients (NS) whereas it decreased slightly (NS) in the controls and in the treated OSAS patients. Pretherapeutic platelet aggregability was significantly lowered by CPAP therapy both at $24: 00 \mathrm{~h}(64.0 \pm 6.5$ versus $55.3 \pm 6.7 \%$, $p<0.05)$ and at $06: 00 \mathrm{~h}(64.1 \pm 6.5$ versus $45.8 \pm 7.6 \% ; \mathrm{p}=0.01)$. Platelet aggregability during sleep in the controls resembled that found in patients with OSAS during CPAP therapy.

The results suggest that obstructive sleep apnoea syndrome contributes, at least in part, to platelet dysfunction and that long-term continuous positive airway pressure treatment may reduce platelet aggregability.

Eur Respir J 2000; 16: 648-652.
*Dept of Medicine I, Ruhr University Bochum, Marienhospital Herne, and "Marienkrankenhous, Kassel, Germany.

Correspondence: B.M. Sanner

Dept of Medicine I

Ruhr University Bochum

Marienhospital

Hoelkeskampring 40

44625 Herne

Germany

Fax: 492323499302

Keywords: Catecholamines

obstructive sleep apnoea syndrome platelet function

sleep

stroke

Received: December 131999

Accepted after revision June 102000
Obstructive sleep apnoea syndrome (OSAS) is a common health burden. It is estimated that $2 \%$ of females and $4 \%$ of males meet the minimal diagnostic criteria for this syndrome [1]. Patients with OSAS suffer from a decreased daytime performance and an impaired quality of life [2]. OSAS has also gained interest because patients are prone to an increased cardiovascular morbidity. Causal relations with systemic hypertension, pulmonary hypertension, coronary artery disease, and right ventricular dysfunction are being discussed [3-8]. There also seems to be an increased risk of myocardial infarction and stroke $[9,10]$ and it is hypothesized that this cardiovascular morbidity is responsible for a reduced cumulative survival rate in OSAS patients [11-13].

Platelets play an important role in the pathogenesis of cardiovascular disease. Blood platelet dysfunction has received attention as a potential cause of increased cardiovascular morbidity not only in essential hypertension but even in apparently healthy middle-aged males $[14,15]$. Since platelet aggregability plays an important role in the pathogenesis of atherosclerotic vasoocclusive disease, the question arises whether an altered platelet aggregability may contribute to the increased cardiovascular morbidity in OSAS patients, too. Therefore, the platelet aggregability in untreated and treated OSAS patients as well as in healthy subjects was measured. The authors found that treatment of OSAS by CPAP decreases platelet aggregability and restores the physiological diurnal pattern of platelet aggregability.

\section{Patients and methods}

Seventeen consecutive male patients with a mean age of $53.0 \pm 2.1$ yrs (range, 35-66 yrs) and a mean body mass index of $32.8 \pm 1.5 \mathrm{~kg} \cdot \mathrm{m}^{-2}$ (range, $25.5-50.5 \mathrm{~kg} \cdot \mathrm{m}^{-2}$ ) were included in the study. They had all been referred to the sleep laboratory for snoring, suspected OSAS, or excessive daytime sleepiness and had significant symptomatic OSAS (Epworth Sleepiness Scale $\geq 10$ ) with an apnoea/hypopnoea index $\geq 10 / \mathrm{h}$ of sleep requiring CPAP therapy. Fifteen male patients with a mean age of $50.1 \pm 3.6 \mathrm{yrs}$ (range, $26-83 \mathrm{yrs}$ ) and a mean body mass index of $24.7 \pm 0.8 \mathrm{~kg} \cdot \mathrm{m}^{-2}$ (range, $20.8-30.9 \mathrm{~kg} \cdot \mathrm{m}^{-2}$ ) without any history of snoring, witnessed apnoea, or hypersomnia served as controls.

Exclusion criteria were the intake of non-steroidal inflammatory drugs or glucocorticoids within 14 days prior to the study day, age $<18$ yrs, smoking or tobacco use, or a known or newly verified coagulation or platelet disorder. Patients with OSAS and craniofacial malformations or other conditions that make CPAP treatment impossible, or with cancer, and those who did not tolerate CPAP therapy, 
in whom CPAP was not effective, or in whom an insufficient compliance to follow-up was to be expected because of either distance or unreliability were also excluded from the study. No patient was receiving sedatives or muscle relaxants. There were no shift workers in the population. A change of medication was not allowed during follow-up.

The study protocol was approved by the local Ethics Committee. Informed consent was obtained from all patients before entry into the study.

\section{Polysomnography}

All patients underwent overnight polysomnography (Somnostar 4100, SensorMedics Co., Yorba Linda, CA, USA) according to widely accepted methods [16]. It consisted of a continuous polygraphic recording from surface leads for electroencephalography, electrooculography, submental and leg electromyography, and electrocardiography, and from noninvasive sensors for nasal and oral airflow (thermistor), tracheal sounds (microphone), thoracic and abdominal respiratory movement (inductance plethysmography), and oxyhaemoglobin level (Sat-Trak $^{\mathrm{TM}}$, finger-pulse oximeter, SensorMedics Co., Yorba Linda, CA, USA). The transducers and lead wires permitted normal positional changes during sleep. Bedtime was between 22:00 h and 24:00 h and awakening time was at $06: 00 \mathrm{~h}$ (time of the last venipuncture). Polysomnography was terminated after final waking. The entire recording was supervised by a technician.

Polysomnography records were scored in 30-s periods for sleep, breathing, and oxygenation. According to the commonly used clinical criteria [17], a breathing event during objectively measured sleep was defined as abnormal if either a complete cessation of airflow lasting $10 \mathrm{~s}$ or more took place (apnoea) or a reduction in respiratory airflow of $50 \%$ or more lasting longer than $10 \mathrm{~s}$ could be discerned (hypopnoea). Obstructive apnoea was defined as absence of airflow in the presence of paradoxical chestwall motion. The average number of episodes of apnoea and hypopnoea per hour of sleep (apnoea/hypopnoea index) was calculated. OSAS was diagnosed when the apnoea/hypopnoea index was $\geq 10 / \mathrm{h}$, associated with typical clinical features. Sleep was staged manually using the method of ReChtSchafFEN and KaLEs [18].

\section{Blood sample collection}

Blood samples were drawn without using a tourniquet from an antecubital vein in the supine position, with use of a 21-gauge needle on the night of polysomnographic evaluation (in patients with OSAS) at 20:00 h, 24:00 h, and 06:00 h. Separate venipunctures were performed at each time point. A 9-mL sample for platelet-aggregation studies was collected in a plastic syringe containing $1 \mathrm{~mL}$ of $3.8 \%$ sodium citrate.

\section{Platelet aggregometry}

The blood was immediately centrifuged for $10 \mathrm{~min}$ at $100 \times g$ to obtain platelet-rich plasma. The platelet-rich plasma was then transferred by pipette to a polystyrene tube, which was tightly capped. Platelet-poor plasma was obtained by centrifuging plasma for $15 \mathrm{~min}$ at $1,500 \times \mathrm{g}$. Platelet concentration was adjusted to $2.5 \times 10^{8}$ cells $\cdot \mathrm{mm}^{2}$ with platelet-poor plasma. Aggregometry was performed according to the method of BORN [19], with use of PAP-4 aggregometer (Moelab, Hilden, Germany), at $37^{\circ} \mathrm{C}$ with $1-\mathrm{mL}$ silicon-treated cuvettes and rotating bars $(1,000$ revolutions per minute). Aliquots of platelet-rich plasma were zeroed against autologous platelet-poor plasma solution so that the platelet-rich plasma gave no light transmittance and the platelet-poor plasma gave 100\% light transmittance. The first aliquot of $0.45 \mathrm{~mL}$ was mixed with a $0.05-\mathrm{mL}$ volume of $1.5 \mathrm{mM} \cdot \mathrm{L}^{-1}$ arachidonic acid to determine whether the subject had taken a nonsteroidal inflammatory drug in the 14 days before the study. Epinephrine, adenosine diphosphate (ADP), and collagen (Bio/Data Corp., Hatboro, PA, USA) were used as aggregating reagents with a final concentration of 10.0 $\mu \mathrm{M}$ (epinephrine and ADP) or $95 \mu \mathrm{g}$ (collagen). To minimize inherent variations in aggregation response, the studies using epinephrine were started $60 \mathrm{~min}$ after venipunctures and were completed within $90 \mathrm{~min}$ of venipuncture.

Data were expressed as the change in optical density or transmittance per minute (slope) and as maximal aggregation (\%). Platelet aggregation may have a primary and a secondary wave. The measurements of slopes were carried out on the primary and secondary wave, and maximal aggregation reflects the intensity of the total wave of aggregation.

\section{Follow-up}

All 17 patients with symptomatic OSAS treated with CPAP were followed for 6 months and then reevaluated by polysomnography and platelet aggregometry. Compliance with prescribed CPAP therapy was based on the actual hours of CPAP usage as registered by an integrated hour meter. Subjective sleepiness was again determined on the Epworth Sleepiness Scale.

\section{Statistical analyses}

Results are given as means \pm SEM. All p-values reported are two-tailed. Statistical analyses were performed with the computer software SPSS for Windows (SPSS, Chicago, IL, USA). Intergroup differences were analysed with Student's t-test for paired or unpaired samples as appropriate, with Bonferroni's correction for multiple comparisons. The relationships between aggregation parameters and the severity of OSAS were explored by univariate regression analyses.

\section{Results}

Out of the 17 patients with OSAS, 9 were being treated for systemic hypertension (7 with angiotensin converting enzyme inhibitors, 4 with calcium channel blockers, 3 with a beta blocking agent) at the time of the study, 1 patient had diabetes mellitus that was treated with insulin. Three 
controls were treated for systemic hypertension (2 with angiotensin converting enzyme inhibitors, 1 with a beta blocking agent). No treatment with medication was stopped for the purpose of this study. Patients with OSAS and the controls did not differ with respect to age, but the body mass index was higher in the OSAS group.

\section{Sleep analysis}

All 17 patients had polysomnographically verified symptomatic OSAS with a mean apnoea/hypopnoea index of $32.2 \pm 4.2 / \mathrm{hr}$ of sleep (table 1 ). Apnoeic episodes were accompanied by mean minimal oxygen saturation of $77.4 \pm 3.6 \%$.

\section{Platelet aggregometry}

Aggregation in vitro was performed with platelets adjusted to $2.5 \times 10^{8}$ cells $\cdot \mathrm{mm}^{2}$. Aggregation induced by arachidonic acid, ADP, and collagen in vitro did not change significantly in the OSAS or control patients from 20:00 h to $24: 00 \mathrm{~h}$ or $06: 00 \mathrm{~h}$, respectively. Likewise, when stimulated in vitro with epinephrine, there was no significant change in overnight aggregability in the OSAS group or in the controls ( $\mathrm{p}=0.08$ ) (fig. 1). In accordance, the slope did not change significantly overnight although it decreased slightly in the controls.

Correlation analyses did not demonstrate a significant relationship between the severity of OSAS and the slope, the degree of aggregation, or the change of aggregation overnight.

\section{Follow-up}

All patients tolerated the treatment well, and none complained of excessive daytime sleepiness with CPAP treatment. The usage of CPAP during the study period, as

Table 1. - Effect of continuous positive airway pressure therapy on body mass index and polysomnographic data

\begin{tabular}{|c|c|c|c|}
\hline & $\begin{array}{l}\text { Before } \\
\text { treatment }\end{array}$ & $\begin{array}{c}\text { After } \\
\text { treatment }\end{array}$ & $\begin{array}{l}\text { Statistical } \\
\text { significance }\end{array}$ \\
\hline $\begin{array}{l}\text { Body mass } \\
\text { index } \mathrm{kg} \cdot \mathrm{m}^{-2} *\end{array}$ & $32.9 \pm 1.6$ & $33.2 \pm 1.6$ & NS \\
\hline Total sleep time min & $401.3 \pm 13.5$ & $346.3 \pm 9.8$ & $<0.01$ \\
\hline Sleep efficiency \% & $91.0 \pm 2.5$ & $85.4 \pm 2.5$ & NS \\
\hline Sleep stage $1+2 \%$ & $66.8 \pm 3.2$ & $60.6 \pm 2.7$ & NS \\
\hline Sleep stage $3+4 \%$ & $13.4 \pm 2.0$ & $15.6 \pm 2.0$ & NS \\
\hline Sleep stage REM** \% & $12.7 \pm 2.6$ & $13.7 \pm 2.1$ & NS \\
\hline $\begin{array}{l}\text { Apnoea/hypopnoea } \\
\text { index } n \cdot h^{-1}\end{array}$ & $32.2 \pm 4.2$ & $3.3 \pm 1.1$ & $<0.01$ \\
\hline$t<90 \% \% \%^{*}$ & $25.4 \pm 8.7$ & $3.2 \pm 2.6$ & $<0.01$ \\
\hline $\begin{array}{l}\text { Minimum nocturnal } \\
\mathrm{S}_{\mathrm{a}, \mathrm{O}_{2}} \%\end{array}$ & $77.4 \pm 3.6$ & $89.0 \pm 1.1$ & $<0.01$ \\
\hline $\begin{array}{l}\text { Epworth Sleepiness } \\
\text { Scale }\end{array}$ & $13.8 \pm 3.0$ & $4.9 \pm 2.2$ & $<0.01$ \\
\hline
\end{tabular}

Values are presented as mean \pm SEM. *: The weight in kilograms divided by the square of the height in meters; **: rapid eye movement; $*$ : percentage of time during sleep with an oxygen saturation $<90 \%$.

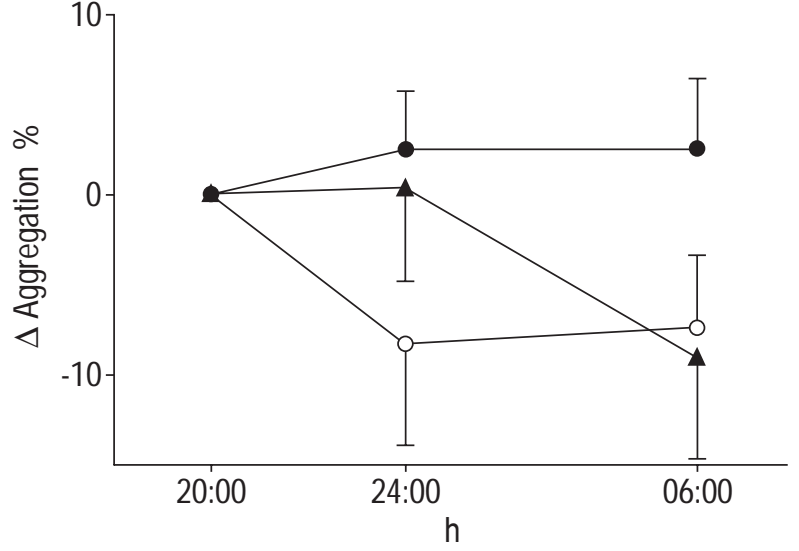

Fig. 1. - Change of percent in vitro platelet aggregation induced by epinephrine at 24:00 $\mathrm{h}$ and 06:00 h compared to 20:00 $\mathrm{h}$ in both control patients and those with obstructive sleep apnoea syndrome (OSAS) on diagnostic nights and with continuous positive airway pressure (CPAP). Increasing aggregability is represented by positive values. Data are presented as mean \pm SEM. The difference between the three groups was not significant $(\mathrm{p}=0.08)$. 0 : OSAS; $\boldsymbol{\Delta}$ : OSAS-CPAP; $\bigcirc$ : controls.

indicated by the hour meter, varied widely with an average of $3.9 \pm 0.6 \mathrm{~h} \cdot$ night $^{-1}$ (range, 0.2-6.2). The mean CPAP pressure was $8.1 \pm 0.7 \mathrm{kPa}$ (range, 4-15). Body mass index did not change significantly during follow-up.
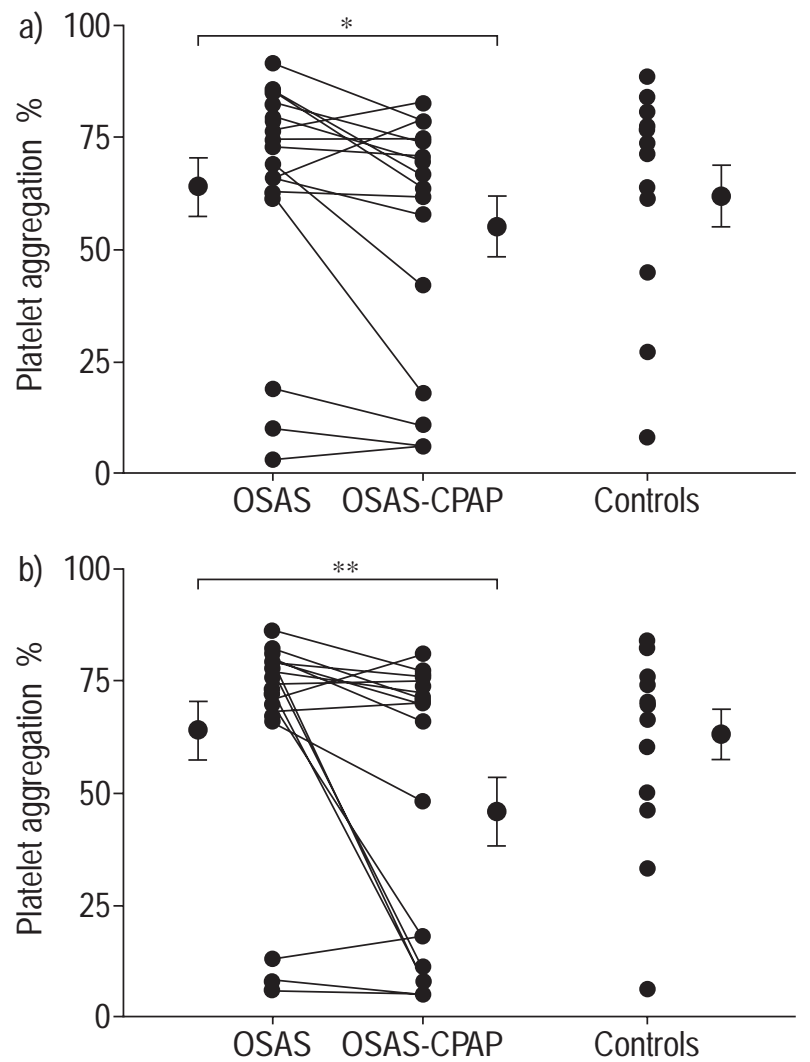

Fig. 2. - Effect of continuous positive airway pressure treatment on platelet aggregation in vitro induced by epinephrine at: a) 24:00 $\mathrm{h}$ and b) at 06:00 $\mathrm{h}$ in patients with obstructive sleep apnoea syndrome (OSAS). There is no significant difference between treated OSAS patients and controls. *: $\mathrm{p}<0.05 ; * *$ : $\mathrm{p}=0.01$. 
CPAP was still effective in treating OSAS in all but one of the patients (table 1). Medication was not changed in the meantime.

Maximal aggregation in vitro induced by epinephrine decreased in 14 and increased in 3 patients, resulting in a significant change in the study group, both at $24: 00 \mathrm{~h}$ $(64.0 \pm 6.5$ versus $55.3 \pm 6.7 \% ; \mathrm{p}<0.05)$ and at $06: 00 \mathrm{~h}(64.1 \pm$ 6.5 versus $45.8 \pm 7.6 \%$; $p=0.01$; fig. 2 ). In accordance, the slope of the 06:00 h sample decreased significantly with CPAP therapy $(17.2 \pm 1.9$ versus $13.8 \pm 2.0 ; \mathrm{p}<0.05)$. One of the patients with an increase in aggregability with CPAP was not treated adequately with CPAP (apnoea/ hypopnoea index at control $13.5 / \mathrm{h}$ of sleep, minimum oxygen saturation during sleep $81 \%$ ), and the two others did not use their device on a regular basis (average CPAP use 0.2 and $0.9 \mathrm{~h} \cdot$ night $^{-1}$, respectively).

Aggregation parameters of OSAS patients treated with CPAP did not differ from those of the controls. A decrease in overnight aggregability could be detected in patients undergoing CPAP therapy, comparable to the control group, but the change was not significant (fig. 1).

Correlation analyses could not document a significant relationship between the change of aggregation parameters and the change of the severity of OSAS as indicated by the apnoea/hypopnoea index or the oxygenation parameters.

\section{Discussion}

The results indicate that in vitro platelet aggregability is altered in patients with OSAS. While it usually decreases overnight, as previously described in healthy males [20] and confirmed in the controls, it increased slightly, although not significantly, in the OSAS patients. The normal diurnal pattern could be restored with long-term therapy of OSAS over 6 months. Furthermore, treatment with CPAP significantly decreased mean platelet aggregability at 24:00 $\mathrm{h}$ and 06:00 $\mathrm{h}$ towards those values found in the controls.

The extent of the in vitro CPAP-induced decrease in aggregability is small, and the measured variables at baseline are generally within the established normal range. Although the exact mechanisms of platelet activation in OSAS are not understood, the factor that is probably most important in these patients is the augmented sympathetic activity with increased concentrations of epinephrine and norepinephrine as a result of hypoxaemia and repetitive arousals from sleep $[5,21]$. Elevated circulating catecholamines cause concentration-dependent platelet activation in vitro and in vivo [22]. The epinephrine-induced platelet responses are more effectively blocked by yohimbine and rauwolscine than by prazosin, indicating that epinephrine stimulates human blood platelets by an $\alpha_{2}$-adrenergic receptor mechanism [23]. Activation of these receptors induces fibrinogen receptor exposure on the platelet surface and fibrinogen binding [24].

The finding in this study of an increased platelet aggregability in patients with OSAS is supported by two studies that measured spontaneous platelet aggregation and activation by flow cytometric determination of P-selectin expression using a monoclonal antibody $[25,26]$.

Why does CPAP treatment over 6 months reduce platelet aggregability? It is known that endurance training lowers plasma concentrations of catecholamines and dec- reases the platelet response to acute exercise [27, 28]. Regular treatment with CPAP may also reduce sympathetic activity in OSAS patients [29], thereby influencing platelet function. In addition, part of the effect may also be attributed to the beneficial effect of lifestyle modification, often associated with hospital treatment, on cardiovascular disease.

The causal relationship between effective treatment of OSAS and reduction of platelet aggregability is furthermore supported by the observation that platelet aggregation increased, apparently in spite of CPAP treatment, only in those 3 patients with OSAS in whom CPAP treatment was ineffective, and who did not use their devices on a regular basis.

A relationship between the degree of platelet dysfunction or variability overnight and the severity of OSAS could not be demonstrated. This may be due to the small study group, or, alternatively, further mechanisms may contribute to alteration in platelet function in patients with OSAS.

It is worth noting that in contrast to the controls, CPAPtreated patients with OSAS had a slightly elevated mean platelet aggregability at 24:00 h. This might be due to the study design with blood sampling in the night of control polysomnography. Mean total sleep time with treatment was less than $6 \mathrm{~h} \cdot$ night $^{-1}$ in the CPAP-treated group, indicating that some of the patients fell asleep only shortly before $24: 00 \mathrm{~h}$ or even after the 24:00 h blood sampling so that in some patients the values represent more the awake than the asleep status.

The authors are well aware that in vitro platelet aggregation does not necessarily represent in vivo changes. But it is known that platelet aggregability as assessed by in vitro methods correlates well with myocardial infarction, sudden death, and long-term incidence of fatal coronary heart disease in a population of apparently healthy middleaged males [15, 20].

A limitation of the conclusions comes from the methodology of blood sampling: as repeated venous punctures were used, platelet aggregation could have been affected indirectly by causing arousals and hence sympathetic nervous activation. However, the authors in this study together with other authors, decided in favour of this methodology and against using a continuously placed catheter, since with the latter procedure an even more profound alteration of platelet function has to be expected. Furthermore, the described differences between treated or untreated patients cannot be invalidated by this methodological problem, since in all patients venous blood sampling was carried out by the same method.

A further limitation of the conclusions is that the control subjects had not been studied polysomnographically. The exclusion of obstructive sleep apnoea by clinical criteria only may not be valid, since a discrepancy between symptoms and polysomnographic findings has been reported.

So far, the impact of an altered platelet function on cardiovascular morbidity and mortality has not been examined in OSAS patients. But it can be speculated that an increased aggregability might be harmful in acute cardiovascular disease, especially with respect to the observation of an augmented apnoeic activity in patients with unstable angina pectoris or acute myocardial infarction $[9,30]$.

To conclude, this study suggests that obstructive sleep apnoea syndrome contributes, at least in part, to platelet 
dysfunction and that long-term continuous positive airway pressure treatment may reduce platelet aggregability.

\section{References}

1. Young T, Palta M, Dempsey J, Skatrud J, Weber S, Badr $\mathrm{W}$. The occurrence of sleep disordered breathing among middle-aged adults. N Engl J Med 1993; 328: 1230-1235.

2. Jenkinson C, Davies RJO, Mullins R, Stradling JR. Comparison of therapeutic and subtherapeutic nasal continuous positive airway pressure for obstructive sleep apnoea: a randomised prospective parallel trial. Lancet 1999; 353: 2100-2105.

3. Brooks D, Horner RL, Kozar LF, Render-Teixeira CL, Phillipson EA. Obstructive sleep apnea as a cause of systemic hypertension. Evidence from a canine model. $J$ Clin Invest 1997; 99: 106-109.

4. Young T, Peppard P, Palta M, et al. Population-based study of sleep-disordered breathing as a risk factor for hypertension. Arch Intern Med 1997; 157: 1746-1752.

5. Ziegler MG, Nelesen R, Mills P, Ancoli-Israel S, Kennedy B, Dimsdale JE. Sleep apnea, norepinephrinerelease rate, and daytime hypertension. Sleep 1997; 20: 224-231.

6. Laks L. Pulmonary arterial pressure in sleep apnea. Sleep 1993; 16: S41-S43.

7. Peker Y, Kraiczi H, Hedner J, Loth S, Johansson A, Bende M. An independent association between obstructive sleep apnoea and coronary artery disease. Eur Respir $J$ 1999; 14: 179-184.

8. Nahmias J, Lao R, Karetzky M. Right ventricular dysfunction in obstructive sleep apnoea: reversal with nasal continuous positive airway pressure. Eur Respir J 1996; 9: 945-951.

9. Hung J, Whitford EG, Parsons RW, Hillman DR. Association of sleep apnoea with myocardial infarction in men. Lancet 1990; 336: 261-264.

10. Partinen M, Palomaki H. Snoring and cerebral infarction. Lancet 1985; II: 1325-1326.

11. He J, Kryger MH, Zorik FJ, Conway W, Roth T. Mortality and apnea index in obstructive sleep apnea. Chest 1988; 94: 9-14.

12. Partinen M, Jamieson A, Guilleminault C. Long-term outcome for obstructive sleep apnea syndrome patients. Chest 1988; 94: 1200-1204.

13. Lindberg E, Janson C, Svärdsudd K, Gislason T, Hetta J, Boman G. Increased mortality among sleepy snorers: a prospective population based study. Thorax 1998; 53: 631-637.

14. Nyrop M, Zweifler AJ. Platelet aggregation in hypertension and the effects of antihypertensive treatment. $J$ Hypertens 1988; 6: 263-269.

15. Thaulow E, Erikssen J, Sandvik L, Stormorken H, Cohn PF. Blood platelet count and function are related to total and cardiovascular death in apparently healthy men. Circulation 1991; 84: 613-617.
16. American Thoracic Society - Medical Section of the American Lung Association. Indications and standards for cardiopulmonary sleep studies. Am Rev Respir Dis 1989; 139: 559-568.

17. American Academy of Sleep Medicine Task Force. Sleeprelated breathing disorders in adults: Recommendations for syndrome definition and measurement techniques in clinical research. Sleep 1999; 22: 667-689.

18. Rechtschaffen A, Kales A. A manual of standardized terminology, techniques and scoring system for sleep stages of human subjects. Washington, DC, U.S. Government Printing Office, 1968 (NIH publication No. 204).

19. Born GV. Aggregation of blood platelets by adenosine diphosphate and its reversal. Nature 1962; 194: 927929.

20. Tofler GH, Brezinski D, Schafer AI, et al. Concurrent morning increase in platelet aggregability and the risk of myocardial infarction and sudden cardiac death. $N$ Engl $J$ Med 1987; 316: 1514-1518.

21. Somers VK, Dyken ME, Clary MP, Abboud FM. Sympathetic neural mechanisms in obstructive sleep apnea. $J$ Clin Invest 1995; 96: 1897-1904.

22. Larsson PT, Wallen NH, Hjemdahl P. Norepinephrineinduced human platelet activation in vivo is only partly counteracted by aspirin. Circulation 1994; 89: 19511957.

23. Grant JA, Scrutton MC. Novel alpha ${ }_{2}$-adrenoceptors primarily responsible for inducing platelet aggregation. Nature 1979; 277: 659-661.

24. Shattil SJ, Budzynski A, Scrutton MC. Epinephrine induces platelet fibrinogen receptor expression, fibrinogen binding, and aggregation in whole blood in the absence of other excitatory agonists. Blood 1989; 73: 150-158.

25. Bokinsky G, Miller M, Ault K, Husband P, Mitchell J. Spontaneous platelet activation and aggregation during obstructive sleep apnea and its response to therapy with nasal continuous positive airway pressure. A preliminary investigation. Chest 1995; 108: 625-630.

26. Eisensehr I, Ehrenberg BL, Noachtar S, et al. Platelet activation, epinephrine, and blood pressure in obstructive sleep apnea syndrome. Neurology 1998; 51: 188-195.

27. Winder WW, Hagberg JM, Hickson RC, Ehsani M, McLane JA. Time course of sympathoadrenal adaptation to endurance exercise training in man. $J$ Appl Physiol 1978; 45: 370-374.

28. Wang JS, Jen CJ, Chen HI. Effects of exercise training and deconditioning on platelet function in men. Arterioscler Thromb Vasc Biol 1995; 15: 1668-1674.

29. Hedner J, Darpö B, Ejnell H, Carlson J, Caidahl K. Reduction in sympathetic activity after long-term CPAP treatment in sleep apnoea: cardiovascular implications. Eur Respir J 1995; 8: 222-229.

30. Moruzzi P, Sarzi-Braga S, Rossi M, Contini M. Sleep apnoea in ischaemic heart disease: differences between acute and chronic coronary syndromes. Heart 1999; 82: 343-347. 